\title{
Companion diagnostics and molecular imaging- enhanced approaches for oncology clinical trials
}

This article was published in the following Dove Press journal:

Drug Design, Development and Therapy

II September 2015

Number of times this article has been viewed

\author{
Ronald L Van Heertum \\ Robert Scarimbolo \\ Robert Ford \\ Eli Berdougo \\ Michael O’Neal \\ BioClinica Inc, Princeton, \\ PA, USA
}

Correspondence: Ronald L Van Heertum BioClinic Inc., 100 Overlook Center, Princeton, NJ 08540, USA

Tel + I 6099362868

Fax +I 6095144964

Email ronald.vanheertum@bioclinica.com

\begin{abstract}
In the era of personalized medicine, diagnostic approaches are helping pharmaceutical and biotechnology sponsors streamline the clinical trial process. Molecular assays and diagnostic imaging are routinely being used to stratify patients for treatment, monitor disease, and provide reliable early clinical phase assessments. The importance of diagnostic approaches in drug development is highlighted by the rapidly expanding global cancer diagnostics market and the emergent attention of regulatory agencies worldwide, who are beginning to offer more structured platforms and guidance for this area. In this paper, we highlight the key benefits of using companion diagnostics and diagnostic imaging with a focus on oncology clinical trials. Nuclear imaging using widely available radiopharmaceuticals in conjunction with molecular imaging of oncology targets has opened the door to more accurate disease assessment and the modernization of standard criteria for the evaluation, staging, and treatment responses of cancer patients. Furthermore, the introduction and validation of quantitative molecular imaging continues to drive and optimize the field of oncology diagnostics. Given their pivotal role in disease assessment and treatment, the validation and commercialization of diagnostic tools will continue to advance oncology clinical trials, support new oncology drugs, and promote better patient outcomes.

Keywords: companion diagnostics, molecular imaging, oncology trials, personalized medicine, diagnostic assays
\end{abstract}

\section{Clinical trial paradigm shift}

Traditional approaches to drug development and clinical trials based on the premise of "one size fits all" are becoming less cost-effective and suboptimal clinically. ${ }^{1}$ This is due mostly to the escalating costs required to develop a new drug, diminishing returns on drug investments, and a high rate of failure for Phase III clinical trials, particularly in oncology drug development. ${ }^{2}$ As a result, the number of new drugs approved by the US Food and Drug Administration (FDA) has remained relatively flat and the number of new drug submissions has decreased significantly over the last decade. ${ }^{3}$ The commitment of the FDA to drive innovation in drug development through its Critical Path Initiative appears to have contributed to a modest uptick in new drug approvals in the last several years.

In line with the FDA initiative to improve the efficiency of drug development and the success rates of late-phase studies, drug sponsors require methodologies that can shorten the length of clinical trial cycles, reduce the number of human subjects required, and provide more reliable early clinical phase assessments for go/no go trial decisions. Many sponsor companies are using companion diagnostic assays and diagnostic imaging studies to help streamline the clinical trial process.

Companion diagnostic assays (also referred to in the literature as pharmacodiagnostics or theranostics) provide a test that can identify the presence or absence of a 
biomarker that is predictive of a patient's phenotype. This approach relies on a detailed understanding of the molecular basis of disease in an individual patient that can subsequently be used to follow-up with a tailored course of treatment based on the presence of specific disease biomarkers. Different classes of biomarkers include somatic mutations, polymorphisms, and gene/protein expression profiles that are associated with a particular disease state. In addition to identifying patients likely to respond to a personalized treatment approach, the incorporation of a diagnostic imaging technique or a diagnostic imaging study in clinical trials allows clinicians and scientists to non-invasively assess the presence, location, and extent of disease for objective, quantitative monitoring of disease progression and response to treatments.

\section{Diagnostic approaches provide key benefits}

Throughout the clinical trial process, the ability to detect and visualize patient biomarkers using companion diagnostic assays and diagnostic imaging tools provides clinicians and drug developers with tools that facilitate faster, safer, and more efficient clinical trials (Figure 1). Early on, they can be used to determine and optimize trial eligibility and enrollment by confirming the presence and quantity of a drug target in an individual patient. During a clinical trial, companion diagnostic assays and diagnostic imaging can be used to monitor and improve treatment responses and patient outcomes by identifying and predicting patient sub-populations that are most likely to respond to a given treatment. Diagnostic approaches not only indicate the presence of a molecular target, but can also inform the off-target effects of a therapeutic, providing increased predictive power for toxicity and adverse effects associated with a drug. Finally, companion diagnostics and diagnostic imaging can inform whether a treatment is reaching its target, providing drug sponsors with an alternative to strict titration studies for determining optimal dosing. Taken together, these approaches are providing new avenues for identifying appropriate patient cohorts for inclusion in a study, monitoring disease, and assessing drug efficacy in individual patients, all of which contribute to potential economic benefits for drug sponsors.

As an example, comparative data from drugs approved for the treatment of non-small cell lung cancer (Xalkori ${ }^{\circledR}$, Pfizer, Inc., New York, NY, USA; and Tarceva ${ }^{\circledR}$, OSI Pharmaceuticals, Inc., Melville, New York, USA and Genentech, Inc., South San Francisco, CA, USA) illustrate the financial benefit of incorporating a companion diagnostic early in the course of drug development. Xalkori, which was codeveloped with a companion diagnostic (Vysis ALK Break Apart fluorescence in situ hybridization probe, Abbott Laboratories, Abbott Park, IL, USA) required approximately threefold fewer patients in clinical trials (960 compared with 3,110), showed an approximately threefold reduction in time from Phase I to approval (1.8 years compared with 5.3 years), and had an overall reduced relative development cost per patient (100\% compared with 154\%). ${ }^{4}$ Furthermore, the addition of epidermal growth factor receptor (EGFR) testing (Hoffman-La Roche Ltd., Basel, Switzerland) as a companion diagnostic for Tarceva in 2013 helped advance the drug to a first-line treatment in a select population and resulted in positive growth forecasts for a drug already on the market for over 8 years. ${ }^{4}$

The importance of diagnostic approaches in drug development is highlighted by the growing global cancer diagnostics market which is expected to reach an estimated value of USD 168.6 billion by the year 2020. ${ }^{5}$ Many newer drugs are being codeveloped with a companion diagnostic assay or imaging diagnostic, sparking the growth of more than 125 companies that provide companion diagnostic products and services. ${ }^{6}$ Additionally, nearly two-thirds of breakthrough therapy designations recently granted by the FDA include a companion diagnostic. ${ }^{7}$ At present, much of the activity in companion diagnostics development is focused in the area of oncology. There are currently 23 companion diagnostics approved by FDA, 22 of which are approved in oncology. ${ }^{8}$ The first companion diagnostic, a HER2 immunohistochemistry assay, was developed in the late 1990s for use with trastuzumab $\left(\right.$ Herceptin $\left.^{\circledR}\right)$, a monoclonal

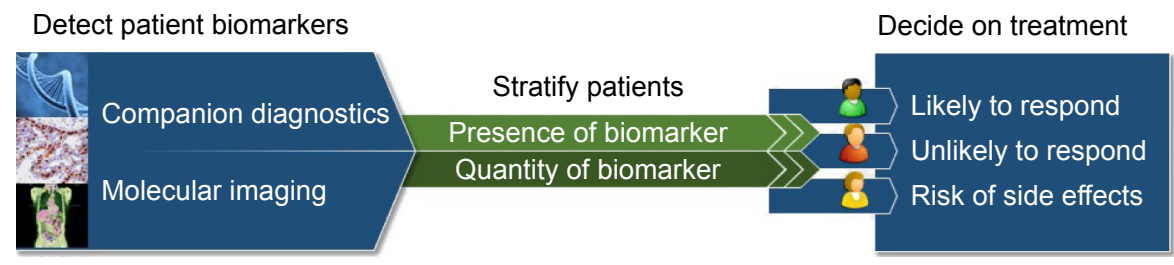

Figure I Companion diagnostics-based treatment strategy for oncology clinical trials.

Notes: In personalized medicine approaches, companion diagnostics (eg, genetic tests, immunohistochemistry, fluorescence in situ hybridization) or molecular imaging (positron emission tomography/single photon emission computed tomography) are used to identify the presence and/or quantity of patient biomarkers. Biomarker data are used to help stratify patients into distinct populations, which helps clinicians decide on a tailored course of therapeutic treatment. 
antibody approved for the treatment of breast cancer. Since then, companion diagnostics have been developed for many targeted oncology therapeutics, including Tarceva, Iressa ${ }^{\circledR}$, Erbitux $^{\circledR}$, and Vectibix ${ }^{\circledR}$ (Table 1). ${ }^{9}$

\section{Advancing oncology trials with diagnostic imaging}

Although companion diagnostic assays continue to improve personalized medicine, there are a number of significant limitations in current diagnostic assay approaches. Specifically, a positive signal generally informs the treating clinician or investigator that a target biomarker is present and, with quantitative assays, to what extent it is present in individual patients. However, the majority of approved diagnostic assays supply very little, if any, information regarding the location and distribution of a target biomarker. In oncology clinical trials, specific knowledge of a target lesion location can be essential, providing accurate biopsy localization and helping to design a treatment plan for tumors involving critical organs (eg, liver, lung, or bone marrow). Another limitation of using companion diagnostics is assay sensitivity (ie, the ability to detect true positives). Yet another limitation of companion diagnostic assays is the relatively narrow scope of biomarker evaluation. Research in the last several years has demonstrated that detection of a therapeutic target is not sufficient to predict drug efficacy and needs to be supplemented by additional data to assess for potential resistance. For example, the presence of KRAS mutations in colorectal cancers expressing EGFR often leads to resistance to anti-EGFR therapy. ${ }^{10}$ Lastly, companion diagnostic assays may require large amounts of tissue samples for the evaluation of multiple biomarkers. This is especially challenging for certain solid tumors where tissue samples may be limited. In such instances, objective assessment by other diagnostic methods is essential for effective use of a companion diagnostic assay.

In clinical oncology studies, diagnostic imaging helps overcome these limitations by providing a reliable methodology to assess the presence, location, and extent of disease in response to treatment. For many years, computed tomography (CT) and magnetic resonance imaging (MRI) have been primary diagnostic imaging tools used for oncology disease assessments. As the use of diagnostic imaging techniques became widespread in clinical trials, a set of standardized imaging assessment criteria from the World Health Organization were established. ${ }^{11}$ In the year 2000, a modified set of criteria called the Response Evaluation Criteria In Solid Tumors (RECIST 1.0) was introduced as part of collaborative efforts between the European Organization for Research and Treatment of Cancer, the National Cancer Institute in the USA, and the National Cancer Institute of Canada

Table I List of companion diagnostics approved by the US Food and Drug Administration

\begin{tabular}{|c|c|c|c|c|}
\hline Drug & Diagnostic & Manufacturer & Indication & Methodology \\
\hline \multirow[t]{3}{*}{ Erbitux, Vectibix } & cobas KRAS Test & Roche Molecular Systems & CRC & RT-PCR \\
\hline & KRAS RGQ Kit & Qiagen & CRC & RT-PCR \\
\hline & EGFR PharmDx Kit & Dako North America & CRC & $\mathrm{IHC}$ \\
\hline Exjade & Ferriscan & Resonance Health Analysis Services & Thalassemia & MRI \\
\hline Gilotrif & EGFR RGQ Kit & Qiagen & NSCLC & RT-PCR \\
\hline Gleevec & C-KIT & Dako North America & GIST & $\mathrm{IHC}$ \\
\hline \multirow[t]{8}{*}{ Herceptin } & INFORM HER2/NEU & Ventana Medical Systems & Breast cancer & $\mathrm{FISH}$ \\
\hline & PATHWAY ANTI-HER-2/NEU & Ventana Medical Systems & Breast cancer & $\mathrm{IHC}$ \\
\hline & INFORM HER2 & Ventana Medical Systems & Breast cancer & Dual color ISH \\
\hline & PATHVYSION HER-2 Kit & Abbott Molecular & Breast cancer & $\mathrm{FISH}$ \\
\hline & INSITE HER-2/NEU Kit & BioGenex Laboratories & Breast cancer & $\mathrm{IHC}$ \\
\hline & SPOT-LIGHT HER2 Kit & Life Technologies & Breast cancer & $\mathrm{CISH}$ \\
\hline & Bond Oracle HER2 System & Leica Biosystems & Breast cancer & $\mathrm{IHC}$ \\
\hline & HER2 Kit & Dako Denmark & Breast cancer & $\mathrm{CISH}$ \\
\hline \multirow{4}{*}{$\begin{array}{l}\text { Herceptin, Perjeta, } \\
\text { Kadcyla }\end{array}$} & HERCEPTEST & Dako Denmark & Breast cancer, Gastric cancer, & $\mathrm{IHC}$ \\
\hline & & & Gastroesophageal adenocarcinoma & \\
\hline & HER2 Kit & Dako Denmark & Breast cancer, Gastric cancer, & $\mathrm{FISH}$ \\
\hline & & & Gastroesophageal adenocarcinoma & \\
\hline Iressa & Therascreen EGFR RGQ Kit & Qiagen & NSCLC & RT-PCR \\
\hline Lynparza & BRACAnalysis & Myriad Genetic Laboratories & Ovarian cancer & PCR \\
\hline Mekinist, Tafinlar & THxID BRAF Kit & bioMérieux & Melanoma & RT-PCR \\
\hline Tarceva & cobas EGFR Test & Roche Molecular Systems & NSCLC & RT-PCR \\
\hline \multirow[t]{2}{*}{ Xalkori } & VYSIS ALK Break Apart Kit & Abbott Molecular & NSCLC & $\mathrm{FISH}$ \\
\hline & ALK (D5F3) Assay & Ventana Medical Systems & NSCLC & $\mathrm{IHC}$ \\
\hline Zelboraf & COBAS 4800 BRAF V600 & Roche Molecular Systems & Melanoma & RT-PCR \\
\hline
\end{tabular}

Abbreviations: EGFR, epidermal growth factor receptor; CRC, colorectal cancer; NSCLC, non-small cell lung cancer; GIST, gastrointestinal stromal tumor; IHC, immunohistochemistry; MRI, magnetic resonance imaging; FISH, fluorescence in situ hybridization; ISH, in situ hybridization; RT-PCR, real-time polymerase chain reaction; $\mathrm{CISH}$, chromogenic in situ hybridization; HER2, human epidermal growth factor receptor; ALK, anaplastic lymphoma kinase; KRAS, kirsten rat sarcoma viral oncogene homolog. 
Clinical Trials Group. ${ }^{12}$ RECIST refined an objective set of criteria that defined when tumor lesions in cancer patients improve (partial or complete response), remain unchanged (stable disease), or worsen (progressive disease) during treatment. Since its introduction, RECIST has been updated (RECIST 1.1) to introduce standards for the assessment of lymph nodes, redefine "measurable" lesions and assessment of disease progression, as well as establish recommendations for standardized image acquisition. ${ }^{13}$ Today, a large number of oncology clinical trials employ RECIST to objectively assess cancer treatment response in solid tumors. Advances in imaging technologies and our understanding of disease have resulted in additional consortia guidelines for standardizing diagnostic imaging in oncology clinical trials. Most notably, the Cheson criteria (1999, 2007, and 2014) have established guidelines for the use of diagnostic imaging using CT, MRI, and fluorodeoxyglucose (FDG)-positron emission tomography (PET) as well as clinical findings for the assessment of lymphoma patients. In addition, the RANO criteria have been established for gliomas, and a number of other criteria have been introduced to specifically assess hepatocellular carcinoma, acute myeloid leukemia, prostate cancer, and the effects of immunotherapies on tumor responses. ${ }^{14-19}$

As these criteria have evolved, it has become clear that conventional anatomical imaging techniques, although very useful, have not supplied all of the objective assessments needed to make accurate early phase go/no go decisions. Initiatives by the Radiological Society of North America, including the Quantitative Imaging Biomarker Alliance, to advance volumetric assessments of tumor lesions continue to gain momentum, and researchers are showing increased interest in developing tools for the evaluation of metrics derived from CT and MRI studies. As an example, techniques such as dual energy CT and spectral CT imaging are being used to better differentiate and characterize certain cancers. These types of image analysis in conjunction with efforts to assess the relationship of CT and MRI to the molecular biology of various tumors, is helping to shape the new fields of radiomics and radiogenomics. Although these approaches hold great potential for oncology clinical trials, it is likely to be several years or more before they can be implemented in a clinical environment.

\section{Molecular imaging diagnostics on the front line}

The field of molecular imaging is rapidly evolving with many different technologies in various stages of development.
At present, nuclear imaging techniques, including PLANAR, single photon emission computed tomography (SPECT), and PET remain the dominant approach for the diagnosis and treatment of cancers. PET and SPECT imaging requires the use of a radiotracer that is injected into a patient prior to interrogating its spatial distribution. PET relies on the detection of gamma photon pairs resulting from the annihilation of positrons (annihilation radiation) originating from a biologically active radiotracer. Using specialized detectors that encircle a patient, (ie, ring scanners) two-dimensional or three-dimensional images of radioactivity distribution within the body can be reconstructed. Similarly, SPECT requires a radiotracer, typically a heavy isotope, and relies on the detection of single gamma photons emitted directly from the radiotracer. SPECT tracers travel in the bloodstream and highlight areas of blood flow. Since SPECT tracers can be imaged at the time of injection, they can be used to detect changes in blood flow to various organs in a variety of disease states. SPECT tracers can also be linked to different biochemical analogs and antibodies to detect tissue specific distribution of cellular targets.

One of the first imaging diagnostic agents used in endocrinology and oncology studies was radiolabeled sodium iodide ( ${ }^{131 \mathrm{NaI}}$ ). This compound has been used effectively to identify individuals with hyperthyroidism, monitor residual thyroid tissue post-surgery, and as a follow-up in treatment for thyroid cancer metastesis. Clinicians routinely rely on other nuclear medicine techniques to identify appropriate patient cohorts likely to respond to treatment and to monitor treatment responses for various cancers. For example, the use of ${ }^{99 \mathrm{~m}}$ Tc-labeled methylene diphosphonate and ${ }^{18} \mathrm{~F}$-labeled $\mathrm{NaF}$ in bone scans to assay for bone metastasis in breast and prostate cancer patients, the use of ${ }^{111}$ In-labeled anti-CD20 antibodies for imaging lymphoid malignancies, and the investigational use of ${ }^{123} \mathrm{I},{ }^{99 \mathrm{~m}} \mathrm{Tc}$, and ${ }^{18} \mathrm{~F}$-labeled prostate-specific membrane antigen (PSMA) for monitoring prostate cancer patients (Table 2).

The ability to probe for molecular targets in cancer patients has opened the door to better, more accurate assessment of disease. Molecular imaging using various PET tracers provides enhanced visualization of tumors, their metabolic activity, and other biological phenotypes (eg, proliferation, hypoxia, expression of target receptors). Furthermore, widespread application of non-invasive imaging like PET/SPECT and gamma scintigraphy, enables the use of many additional tracers in oncology clinical trials (Table 2). For example, ${ }^{18}$ F-FDG and ${ }^{18}$ F-FLT are used to monitor glycolytic activity and proliferation of tumors, 
Table 2 Commonly used radiotracers in PET or SPECT studies

\begin{tabular}{ll}
\hline Tracer & Application \\
\hline$[I 8] \mathrm{F}$ & Imaging bone, measuring glucose metabolism, cell proliferation \\
{$[123] \mathrm{I}$} & Detection and management of thyroid cancer \\
{$[13 \mathrm{I}] \mathrm{I}$} & Detection and management of thyroid cancer \\
{$[\mathrm{III}] \mathrm{ln}$} & Neuroendocrine tumors, lymphomas \\
{$[64] \mathrm{Cu}$} & Hypoxia imaging, tumor receptor targeting \\
{$[\mathrm{II}] \mathrm{C}$} & Diagnosis and monitoring of cancer \\
{$[99 \mathrm{~m}] \mathrm{Tc}$} & Monitoring prostate cancer \\
{$[20 \mathrm{I}] \mathrm{TI}$} & Cardiovascular imaging, brain tumors \\
{$[67] \mathrm{Ga}$} & Detection of lymphomas \\
{$[68] \mathrm{Ga}$} & Tumor imaging, leukocyte-derived malignancies \\
\hline
\end{tabular}

Abbreviations: PET, positron emission tomography; SPECT, single photon emission computed tomography.

respectively, and technetium ${ }^{99 \mathrm{~m}} \mathrm{Tc}$-labeled antibody and peptide compounds are routinely used to label tumors and diagnose sites of cancer.

The elucidation and validation of novel oncology targets using high throughput screens is opening the door to development of potent and selective antibodies and other molecules capable of targeting tumor-specific or tumor-enriched receptors. ${ }^{20}$ These agents can be linked to radionuclides, fluorophores, or other imaging probes (eg, $\left.{ }^{111} \mathrm{In},{ }^{99 \mathrm{~m}} \mathrm{Tc}\right)$ to confirm the presence and anatomical location of cellular targets or developed as cytotoxic therapeutics $\left(e g,{ }^{90} \mathrm{Y},{ }^{131} \mathrm{I}\right)$. Several well studied proteins serve as oncology targets for imaging diagnostics including PSMA, the estrogen receptor (ER), and the folate receptor (Table 3). PSMA is a protein amplified on the surface of nearly all prostate cancer cells and is a validated target for the detection of primary and metastatic prostate cancer. Radiolabeled small molecules targeting PSMA are well tolerated tools for the detection of metastatic prostate cancer. A number of academic centers and pharmaceutical companies are developing and testing molecules labeled with ${ }^{18} \mathrm{~F},{ }^{99 \mathrm{~m}} \mathrm{Tc}$, and ${ }^{123} \mathrm{I}$ that specifically target PSMA. Molecules capable of targeting ER are proving to be extremely valuable for improving breast cancer treatment. Several studies have shown that $16 \alpha-{ }^{18} \mathrm{~F}-$ fluoro-17 $\beta$-estradiol $\left({ }^{18} \mathrm{~F}\right.$-FES $)$, an ER-specific PET tracer, can reliably detect ER-positive tumor lesions and that its uptake correlates well with immunohistochemical scoring for the presence of ER. ${ }^{21-23}$ Currently, ${ }^{18} \mathrm{~F}$-FES is being evaluated in breast cancer patients to determine patient ER status, to help differentiate between benign and malignant lesions, and to differentiate between metastases originating from different tumor types. ${ }^{24}$ Similarly, the folate receptor is overexpressed in many cancer tissues and represents a target for selectively imaging and delivering therapeutics to cancer cells. A companion imaging diagnostic $\left({ }^{99 \mathrm{~m}} \mathrm{Tc}\right.$-labeled folate-targeted molecule) has already been developed to identify tumors that overexpress the folate receptor, and clinical data have shown that patients with metastases that are positive for the folate receptor benefit from treatment with the corresponding folate-targeted small molecule drug conjugate. ${ }^{25,26}$ In addition to showcasing the importance of targeted imaging agents for oncology treatment, this approach provides a paradigm for the codevelopment of an imaging diagnostic and a therapeutic agent and may

Table 3 Key oncology targets for which there are molecular imaging diagnostics

\begin{tabular}{|c|c|c|c|}
\hline Target & Description & Diagnostic & Clinical utility \\
\hline PSMA & Overexpressed in prostate cancer & ${ }^{99 \mathrm{~m} T m-P S M A},{ }^{123}$ I-PSMA & $\begin{array}{l}\text { Detection of primary and metastatic } \\
\text { prostate cancer }\end{array}$ \\
\hline ER & Overexpressed in breast cancer & $16 \alpha-{ }^{18} \mathrm{~F}$-fluoro- $17 \beta$-estradiol & $\begin{array}{l}\text { Determines patient's ER status, } \\
\text { differentiates between benign and } \\
\text { malignant lesions, and differentiates } \\
\text { between metastases originating from } \\
\text { different tumor types }\end{array}$ \\
\hline Folate receptor & Overexpressed in various cancers & ${ }^{99 \mathrm{~m}} \mathrm{Tc}$-labeled folate-targeted molecule & $\begin{array}{l}\text { Identify patients with metastases that } \\
\text { stand to benefit from treatment }\end{array}$ \\
\hline
\end{tabular}

Abbreviations: ER, estrogen receptor; PSMA, prostate-specific membrane antigen. 
contribute to the design of more efficient drug development workflows in the future.

The emergence and development of new SPECT and PET radiopharmaceuticals, and the use of hybrid imaging modalities such as SPECT/CT, PET/CT, or PET/MRI (which combine anatomic imaging with physiological imaging in a single device and enable the acquisition of coregistered anatomical and physiological scans) provide a more complete picture of a patient's disease state. In this respect, the use of molecular imaging is helping to modernize recommendations for the evaluation, staging, and response assessments of cancer patients. As an example, the Cheson criteria was recently revised to require ${ }^{18} \mathrm{~F}$-FDG assessment as the dominant imaging technique for evaluation of FDGavid lymphomas. ${ }^{27}$

Significant improvements in hardware for PET and SPECT imaging, primarily driven by the oncology market, continue to advance widespread application of molecular imaging. Modern day PET and SPECT scanners increasingly are using newer crystal detector materials and solid state photon detectors that are smaller in size, provide increased sensitivity, and have better spatial resolution. New collimator designs and specialized gantries help reduce imaging time and radiation doses, thereby increasing patient safety and comfort. Additionally, newer image reconstruction techniques and software incorporate iterative reconstruction, time-of-flight data, and resolution recovery, which results in improved image contrast, image resolution, and reduce image noise. ${ }^{28}$

The value of molecular imaging approaches for drug development has also led to the design and widespread integration of PET and SPECT techniques in preclinical animal models of disease. Small animal micro-PET and micro-SPECT imaging systems (as well as small-scale anatomical and hybrid imaging systems) are commercially available and are being used in the early drug discovery process to monitor drug toxicity and efficacy in efforts to advance the most promising oncology candidate drugs to human clinical trials. ${ }^{29}$

\section{Molecular imaging gets quantitative}

Quantitative approaches using molecular imaging are extremely valuable as they provide insight as to what is occurring at the cellular level and are often predictive of a tumor response before anatomical changes can be observed. The introduction, validation, and use of quantitative molecular imaging continues to drive and optimize the field of imaging diagnostics. In addition to identifying the presence, location, and distribution of a specific tumor biomarker, radiopharmaceuticals can be used to objectively obtain quantitative measurements, including region of interest assessments of single or multiple areas. Most clinical trials that use molecular imaging rely on relative or semiquantitative approaches, since absolute quantitation methods using radionuclides are very complex and impractical for routine clinical studies. A common measurement used in molecular imaging for assessing treatment responses is the standardized uptake value (SUV). The SUV represents the ratio of the concentration of radioactivity in a selected region to the total injected dose of radioactivity distributed evenly throughout a patient's body. SUV measurements can be calculated as a mean value $\left(\mathrm{SUV}_{\text {mean }}\right)$ or as a maximum value $\left(\mathrm{SUV}_{\max }\right)$, and can be further normalized to a patient's lean body mass or whole body mass. Additional treatment response information can be gained by quantitative assessment of the changing pattern of uptake at multiple different time points (Figure 2).

Other quantitative measurements used in clinical trials include glycolytic index determination, which is a measure of the total metabolic activity of a specific targeted area (eg,

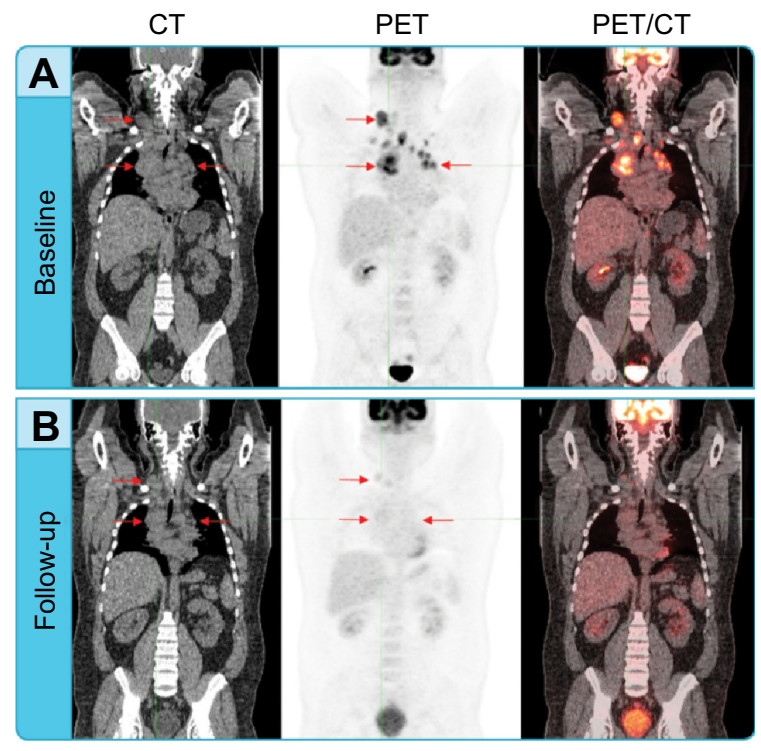

Figure 2 Assessing treatment response using PET and CT.

Notes: (A) Baseline coronal CT, PET, and fused PET/CT images of a patient with lymphoma. Multi-focal bilateral FDG-avid adenopathy, including a large right superior mediastinal mass lesion (arrows) with marked focal FDG uptake visible on the coregistered FDG-PET consistent with lymphoma. (B) Follow-up coronal CT, PET, and PET/CT images of the same patient after two cycles of therapy. The multifocal adenopathy including the large right superior mediastinal mass is still visible on the CT image and the right mediastinal lesion appears stable (arrows). The PET image demonstrates complete resolution of tumor metabolic activity. All previous FDG-avid regions are indiscernible from background, consistent with a CMR. The CMR noted on the PET examination indicates a treatment response before any change is visible by CT.

Abbreviations: CMR, complete metabolic response; CT, computed tomography; FDG, fluorodeoxyglucose; PET, positron emission tomography. 
target tumor lesion) and the standardized uptake peak value $\left(\mathrm{SUV}_{\text {peak }}\right)$, currently used with the Positron Emission Tomography Response Criteria in Solid Tumors (PERCIST). ${ }^{30}$ For longitudinal studies, dynamic measurements acquired over time can be used to generate time/activity curves. In particular, dynamic time/activity curve analysis is useful for assessing diagnostic probes, including their ability to localize in a specific tumor site and duration or residence time in a target site. This type of data can be very useful for determining optimal dosing using the therapeutic equivalent of an imaging companion diagnostic.

Although molecular imaging offers a wide spectrum of applications in drug development and clinical trials, there is only a single imaging companion diagnostic approved by the FDA, called FerriScan ${ }^{\circledR}$. FerriScan uses MRI to select patients and manage therapy for non-transfusiondependent thalassemia. The lack of additional FDA-approved imaging companion diagnostics highlights the opportunity and need for additional agents to be adapted, tested, and validated as diagnostic assays. In order for a molecular imaging test to become an integral part of any clinical trial investigation, the specific molecular imaging study has to be validated in prior investigations as an integrated component of a prospective analysis where it is not utilized to direct treatment decisions. Upon validation, a molecular imaging test can be used as an integral component of clinical trials and may even be required by regulatory agencies in clinical cases prior to and/or following drug administration. ${ }^{31}$ As an example, ${ }^{18}$ FDG-PET imaging is being used in lymphoma clinical trials in conjunction with the Lugano criteria. ${ }^{26}$ Given their pivotal role in clinical trials, it is likely that we will see an increase in the number of imaging companion diagnostics and integrated molecular imaging studies for oncology clinical trials. Even in cases where an imaging companion diagnostic is not incorporated into a clinical trial paradigm, it is important to recognize that the combination of a companion diagnostic assay with the appropriate imaging diagnostic can supply complementary information that cannot be ascertained from either methodology alone.

\section{Companion diagnostics and regulatory requirements}

The importance of companion diagnostics for current and future pharmacotherapy has attracted the attention of global regulatory agencies. For new therapies requiring the use of a diagnostic to qualify patient populations, companion diagnostics must meet typical design control and submission requirements to ensure safety and efficacy. As a result, regulatory agencies are increasing their visibility and offering more structured platforms for diagnostic companies to interact with them.

The FDA has taken significant steps in the last decade to define the companion diagnostic pathway. The FDA has published a drug-diagnostic codevelopment concept paper and created a personalized medicine group within the Office of In Vitro Diagnostics and Radiological Health. Last year, the FDA released a formal guidance called "In vitro companion diagnostic devices", indicating regulatory pathways and requirements for companion diagnostic devices and therapeutic products. ${ }^{32}$ The guidance defines in vitro companion diagnostic devices (IVDs), informs industry and FDA staff on premarket regulatory pathways and enforcement policies, and describes regulatory approval requirements relevant to therapeutic product labeling. In the case of clinical trials, where companion diagnostic assays are used to inform treatments, there are stringent requirements for submission of an investigational device exemption, usually as part of an investigational new drug application (IND). Given that the majority of companion diagnostic assays are considered high-risk devices (class III), there is also a requirement for a premarket approval application.

Although there are a number of therapeutic drugs that require companion diagnostic testing in the EU, the European Medicines Agency has been less transparent regarding companion diagnostics. The IVD Directive 98/79/EC regulates in vitro diagnostic medical devices in the EU and IVD devices require a $\mathrm{CE}$ mark to indicate compliance. Currently, any companion diagnostic entering the EU market is classified as a low-risk device based on CE marking by the manufacturer (self-certification). This results in a major divergence in the approval process between the USA and the EU. There are major changes underway in IVD legislation, including regulation that will provide a single regulatory framework for all EU member states. Under a new draft guidance which entered Parliament last year, companion diagnostics will be assigned as class $\mathrm{C}$ devices, requiring design examination certification by a Notified Body.

The process of achieving regulatory approval for new diagnostic imaging agents also remains extremely challenging, highlighted by the fact that only a handful of new radiotracers have received FDA approval in the last decade. ${ }^{33}$ Although radiotracers are typically administered at doses that are orders of magnitude lower than therapeutics and are designed to measure molecular processes rather than modify them, they are regulated as though they carry the equivalent risk of a therapeutic. In fact, the commercial 
development of a new imaging agent shares many of the same challenges as therapeutic drug development, including target validation, lead selection, establishing high affinity and uptake, achieving adequate clearance, and demonstrating low toxicity. ${ }^{2}$

The FDA has issued a three-part guidance in 2004 surrounding the regulatory pathway to the commercialization of new imaging agents, which covers safety assessments, clinical indications, and the design, analysis, and interpretation of clinical studies. ${ }^{34-36}$ In an effort to facilitate the regulatory process for imaging diagnostics, the FDA has established an exploratory IND for therapeutics and diagnostics, which provides an early look at the distribution and metabolism of new tracers in a small number of patients using early human screening and microdosing experiments. Imaging tracers that show promising results can proceed through traditional clinical trial phases and the filing of a formal IND. The exploratory IND process covers safety and efficacy for measuring a molecular process, but falls short in providing approval for larger clinical trials. To help overcome this, the Society of Nuclear Medicine has put forth a two-step approval process (safety and efficacy in measuring a molecular process and clinical utility and efficacy) specifically for diagnostic imaging agents. ${ }^{37}$ More recently, the Society of Nuclear Medicine created the Molecular Imaging Clinical Trials Network with the use of centralized INDs for non-proprietary radiolabeled tracers to facilitate access to investigational molecular imaging radiopharmaceuticals for clinical trials. The European Medicines Agency has also issued a formal guidance document that outlines the qualification process for biomarker development (EMEA/CHMP/SAWP/72894/2008). ${ }^{38}$ This guidance, updated in 2014, outlines the scientific pathway leading to either a Committee for Medicinal Products for Human Use qualification opinion or qualification advice on innovative methods or drug development tools.

\section{Closing thoughts}

In vitro companion diagnostic assays and in vivo molecular diagnostic imaging continue to advance the field of personalized medicine and are changing the way in which clinicians are treating cancer and other human diseases. Assays and imaging agents are being developed alongside therapeutics to stratify patients and maximize the potential treatment benefit of new oncology therapeutics. These approaches are not only changing the landscape of clinical trials, but are also contributing to important changes in drug development and treatment. With the discovery of new oncology targets and imaging tracers comes increased capabilities to probe, monitor, and evaluate cancer on a molecular level. It is clear that more widespread implementation of imaging diagnostic tools will advance oncology clinical trials and help support new drug approvals in this rapidly expanding therapeutic area.

\section{Disclosure}

RVH, MO, RS, and EB are full-time employees of BioClinica and RF is a consultant for BioClinica. The authors report no other conflicts of interest in this work.

\section{References}

1. DiMasi JA, Hansen RW, Grabowski HG. The price of innovation: new estimates of drug development costs. $J$ Health Econ. 2003;22: $151-185$.

2. Kola L, Landis J. Can the pharmaceutical industry reduce attrition rates? Nat Rev Drug Discov. 2004;3:711-716.

3. Hay M, Thomas DW, Craighead JL, Economides C, Rosenthal J. Clinical development success rates for investigational drugs. Nat Biotechnol. 2014;32:40-51.

4. Agarwal A, Ressler D, Snyder G. The current and future state of companion diagnostics. Pharmgenomics Pers Med. 2015;8:99-110.

5. Transparency Market Research. Cancer diagnostics market (tumor biomarker tests, imaging, endoscopy and biopsy) - global industry analysis, size, share, growth, trends and forecast, 2014-2020. Available from: http://www.transparencymarketresearch.com/cancer-diagnosticsmarket.html. Accessed July 20, 2015.

6. Naylor S, Cole T. Overview of companion diagnostics in the pharmaceutical industry. Drug Discovery World. 2010. Available from: http:// www.ddw-online.com/personalised-medicine/p92845-overview-ofcompanion-diagnostics-in-the-pharmaceutical-industry.spring-10.html. Accessed July 20, 2015.

7. Varond AJ. Trends in personalized medicine. Regulatory Focus. Regulatory Affairs Professional Society; 2013. Available from: http://www. raps.org/WorkArea/DownloadAsset.aspx?id=5275.

8. Nicolaides NC, O'Shannessy DJ, Albone E, Grasso L. Co-development of diagnostic vectors to support targeted therapies and theranostics: essential tools in personalized cancer therapy. Front Oncol. 2014;4:141.

9. US Food and Drug Administration. List of cleared or approved companion diagnostic devices (in vitro and imaging tools). Available from: fda.gov/MedicalDevices/ProductsandMedicalProcedures/ InVitroDiagnostics/ucm301431.htm. Accessed March 27, 2015.

10. Douillard JY, Oliner KS, Siena S, et al. Panitumumab-FOLFOX4 treatment and RAS mutations in colorectal cancer. N Engl J Med. 2013; 369:1023-1034.

11. Miller AB, Hoogstraten B, Staquet M, Winkler A. Reporting results of cancer treatment. Cancer. 1981;47:207-214.

12. Therasse P, Arbuck SG, Eisenhauer EA, et al. New guidelines to evaluate the response to treatment in solid tumors (RECIST guidelines). J Natl Cancer Inst. 2000;92:205-216.

13. Eisenhauer EA, Therasse P, Bogaerts J, et al. New response evaluation criteria in solid tumours: revised RECIST guideline (version 1.1). Eur J Cancer. 2009;45:228-247.

14. Cheson BD, Greenberg PL, Bennett JM, et al. Clinical application and proposal for modification of the International Working Group (IWG) response criteria in myelodysplasia. Blood. 2006;108:419-425.

15. Scher HI, Halabi S, Tannock I, et al. Design and end points of clinical trials for patients with progressive prostate cancer and castrate levels of testosterone: recommendations of the Prostate Cancer Clinical Trials Working Group. J Clin Oncol. 2008;26:1148-1159.

16. Wolchok JD, Axel Hoos A, O'Day S, et al. Guidelines for the evaluation of immune therapy activity in solid tumors: immune-related response criteria. Clin Cancer Res. 2009;15:7412-7420. 
17. Cheson BD, Bennett JM, Kopecky KJ, et al. Revised recommendations of the international working group for diagnosis, standardization of response criteria, treatment outcomes, and reporting standards for therapeutic trials in acute myeloid leukemia. J Clin Oncol. 2003;21: 4642-4649.

18. Lencioni R, Llovet JM. Modified RECIST (mRECIST) assessment for hepatocellular carcinoma. Semin Liver Dis. 2010;30:52-60.

19. van den Bent MJ, Wefel JS, Schiff D, et al. Response assessment in neuro-oncology (a report of the RANO group): assessment of outcome in trials of diffuse low-grade gliomas. Lancet Oncol. 2011;12:583-593.

20. Hoelder S, Clarke PA, Workman P. Discovery of small molecule cancer drugs: successes, challenges and opportunities. Mol Oncol. 2012;6: $155-176$.

21. McGuire AH, Dehdashti F, Siegel BA, et al. Positron tomographic assessment of 16 alpha-[18F] fluoro-17 beta-estradiol uptake in metastatic breast carcinoma. J Nucl Med. 1991;32:1526-1531.

22. Mintun MA, Welch MJ, Siegel BA, et al. Breast cancer: PET imaging of estrogen receptors. Radiology. 1988;169:45-48.

23. Peterson LM, Mankoff DA, Lawton T, et al. Quantitative imaging of estrogen receptor expression in breast cancer with PET and $18 \mathrm{~F}$ fluoroestradiol. J Nucl Med. 2008;49:367-374.

24. van Kruchten M, Glaudemans AW, de Vries EF, et al. PET imaging of estrogen receptors as a diagnostic tool for breast cancer patients presenting with a clinical dilemma. J Nucl Med. 2012;53:182-190.

25. Dosio F, Milla P, Cattel L, et al. EC-145, a folate-targeted vinca alkaloid conjugate for the potential treatment of folate receptor-expressing cancers. Curr Opin Investig Drugs. 2010;11:1424-1433.

26. Leamon CP. Folate-targeted drug strategies for the treatment of cancer. Curr Opin Investig Drugs. 2008;9:1277-1286.

27. Cheson BD, Fisher RI, Barrington SF, et al. Recommendations for initial evaluation, staging, and response assessment of Hodgkin nonHodgkin lymphoma: the Lugano classification. J Clin Oncol. 2014;32: 3059-3068.

28. Slomka PJ, Pan T, Berman DS, Germano G. Advances in SPECT and PET hardware. Prog Cardiovasc Dis. 2015;57:566-578.
29. Jang B. MicroSPECT and microPET imaging of small animals for drug development. Toxicol Res. 2013;29:1-6.

30. Wahl RL, Jacene H, Kasamon Y, Lodge MA. From RECIST to PERCIST: evolving considerations for PET response criteria in solid tumors. J Nucl Med. 2009;50:122S-150S.

31. Mankoff DA, Pryma DA, Clark AS, et al. Molecular imaging biomarkers for oncology clinical trials. J Nucl Med. 2014;55:525-528.

32. US Food and Drug Administration. In vitro companion diagnostic devices. 2014. Available from: http://www.fda.gov/downloads/ MedicalDevices/DeviceRegulationandGuidance/GuidanceDocuments/ UCM262327.pdf. Accessed July 20, 2015.

33. Agdeppa ED, Spilker ME. A review of imaging agent development. AAPS J. 2009;11:286-299.

34. US Food and Drug Administration. Guidance for industry developing medical imaging drug and biological products. Part 1: Conducting Safety Assessments. 2004. Available from: http:/www.fda.gov/downloads/ Drugs/.../Guidances/ucm071600.pdf. Accessed July 20, 2015.

35. US Food and Drug Administration. Guidance for industry developing medical imaging drug and biological products. Part 2: Clinical indications. 2004. Available from: http://www.fda.gov/downloads/Drugs/ GuidanceComplianceRegulatoryInformation/Guidances/ucm071603. pdf. Accessed July 20, 2015.

36. US Food and Drug Administration. Guidance for industry developing medical imaging drug and biological products. Part 3: Design, analysis, and interpretation of clinical studies. 2004. Available from: http://www. fda.gov/downloads/drugs/guidancecomplianceregulatoryinformation/ guidances/ucm071604.pdf. Accessed July 20, 2015.

37. McEwan A, Van Brocklin H, Divgi C. Action plan for emerging molecular imaging technologies. J Nucl Med. 2008;49:37N-40N

38. European Medicines Agency. Qualification of novel methodologies for drug development: guidance to applicants. 2014. Available from: http:// www.ema.europa.eu/docs/en_GB/document_library/Regulatory_and_ procedural_guideline/2009/10/WC500004201.pdf. Accessed July 20, 2015 .

\section{Publish your work in this journal}

Drug Design, Development and Therapy is an international, peerreviewed open-access journal that spans the spectrum of drug design and development through to clinical applications. Clinical outcomes, patient safety, and programs for the development and effective, safe, and sustained use of medicines are a feature of the journal, which has also been accepted for indexing on PubMed Central. The manuscript management system is completely online and includes a very quick and fair peer-review system, which is all easy to use. Visit http://www.dovepress.com/testimonials.php to read real quotes from published authors. 